\title{
Universe consisting of diffusive dark fluids: thermodynamics and stability analysis
}

\author{
Subhayan Maity ${ }^{\mathrm{a}}$, Pritikana Bhandari ${ }^{\mathrm{b}}$, Subenoy Chakraborty ${ }^{\mathrm{c}}$ \\ Department of Mathematics, Jadavpur University, Kolkata, West Bengal 700032, India
}

Received: 6 December 2018 / Accepted: 15 January 2019 / Published online: 29 January 2019

(C) The Author(s) 2019

\begin{abstract}
The present work deals with homogeneous and isotropic FLRW model of the Universe having a system of non-interacting diffusive cosmic fluids with barotropic equation of state (constant or variable equation of state parameter). Due to diffusive nature of the cosmic fluids, the divergence of the energy momentum tensor is chosen to be proportional to the diffusive current. The thermodynamic stability analysis of individual fluids is done and the stability conditions are expressed as restrictions on the equation of state parameter.
\end{abstract}

\section{Introduction}

The greatest challenge of standard cosmology today is to accommodate the recent observational predictions [1-9]. The modern cosmology is facing the challenging issue of explaining the present accelerated expansion of the universe. In the framework of Einstein gravity, cosmologists are speculating some hypothetical exotic matter (known as dark energy (DE) having large negative pressure) to explain this accelerating phase. It is estimated [10] that about $70 \%$ of the cosmic fluid consists of this unknown DE component. The simplest as well as the common candidate for this dark fluid is the cosmological constant (the zero point energy of the quantum fields). Although a large number of available observational data are in support of this cosmological parameter as a DE candidate but there are severe drawback of it at the interface of Cosmology and Particle Physics: the cosmological constant problem [11,12] and the coincidence problem [13]. As a result there are several alternative proposed dynamical DE models into the picture. These DE models have been studied for the last several years [14], yet the cosmological constant is still the best observationally supported DE candidate. However, these different dynamic DE models cannot

\footnotetext{
${ }^{a}$ e-mail: maitysubhayan@gmail.com

be-mail: pritikanab@gmail.com

c e-mail: schakraborty.math@gmail.com
}

be compared from observational view point as these models try to adjust the data seamlessly. As a result, cosmologists have been trying with interacting DE model to have a better understanding of the mechanism of this cosmic acceleration.

From recent past, interacting dark fluid models have been receiving much attention as they can provide small value of the cosmological constant and due to their ability of explaining the cosmic coincidence problem [15-17]. Moreover, recent observed data [18-23] favor interacting dark fluid models and it is possible to have an estimate of the coupling parameter in the interaction term by various observations [21-30]. Further,the cosmologists are of the opinion that these interacting dark fluid models may have the solution to the current tensions on $\sigma_{8}$ and the local value of the Hubble constant $\left(H_{0}\right)$ [22-31]. Moreover, it is speculated that cosmological perturbation analysis may be affected by the interaction terms and consequently, the lowest multipoles of the CMB spectrum [32,33] should have an imprint of it.

On the other hand, the unknown nature of DE may have some clue from the thermodynamic laws which are applicable to all types of macroscopic systems and are based on experimental evidence. However, unlike classical mechanics or electromagnetism, thermodynamical analysis can not predict any definite value for observables, it may only give limit on physical processes. So it is reasonable to believe that thermodynamical study of dark cosmic fluid may indicate some unknown character of it. Investigation in this direction has been initiated recently by Barboza et al. [34]. But their stability conditions demand that the DE should have constant ( - ve) equation of state and it is not supported by observation.

Subsequently, Chakraborty and collaborators [35-38] in a series of works have shown the stability criteria for different type of dark fluids and have presented the stability conditions (in tabular form) for different ranges of the equa- 
tion of state parameter. The present work is an extension of the interacting dark fluid model. Here a particular realization of the non-conservation of the energy momentum tensor is used with a diffusion of dark matter in a fluid of dark energy. The change of the energy-density is proportional to the particle density. The resulting non-equilibrium thermodynamics is studied and stability conditions are explicitly determined. The paper is organized as follows: Sect. 2 deals with thermodynamical analysis of non-interacting cosmic fluids with constant equation of state. The stability criteria for noninteracting cosmic fluids with variable equation of state has been organized in Sect. 3 . Finally, the paper ends with a brief discussion and the conclusion from the result in dark energy range in Sect. 4.

\section{Thermodynamic analysis of non-interacting diffusive cosmic fluids having constant equation of state parameter}

The universe is assumed to have different types of noninteracting cosmic fluids (including dark energy and dark matter). Here it will be examined whether all kinds of diffusive fluids are thermodynamically stable or not in an adiabatic universe . Let the equation of state of these cosmic fluids are barotropic in nature having explicit form (for ith fluid)

$p_{i}=w_{i} \rho_{i}$,

where $\omega_{i}$, a constant, is the barotropic index of the fluid and $p_{i}$ and $\rho_{i}$ are the pressure and energy density of the fluid respectively. So the Friedmann equations for the whole system take the form

$$
\begin{aligned}
& 3 H^{2}=\sum_{i} \rho_{i} \\
& 2 \dot{H}+3 H^{2}=-\sum_{i} p_{i}
\end{aligned}
$$

where $H=a^{-1} \dot{a}$ is the Hubble parameter, $a(t)$ is called the scale factor of the universe as all physical distance is scaled with same factor " $a$ " due to homogeneity and isotropy of space-time. Now due to the diffusive nature of the fluids, they do not obey the matter conservation equation $\left(T_{i}^{\mu \nu}{ }_{; \mu}=0\right)$, rather they follow,

$T_{i}^{\mu \nu} ; \mu=3 k^{2} N_{i}^{v}$,

where $N_{i}^{v}$ is the current of diffusion corresponding to that fluid and $k$ is a constant. But for the whole universe, the total stress-energy tensor is conserved i.e. $\left(\sum_{i} T_{i}^{\mu \nu} ; \mu=0\right)$

$\sum_{i} N_{i}^{v}=0$
In simplest form, the conservation equation (non conservation) takes the form for FLRW universe [39-41] as,

$\frac{\partial \rho}{\partial t}+3 H\left(1+\omega_{i}\right) \rho_{i}=\gamma_{i} a^{-3}$

where, $\gamma_{i}$ is a constant for a particular fluid but it is different for different fluids with $\sum_{i} \gamma_{i}=0$. Now, in the context of classical thermodynamics, the contribution to the energy of the universe by ith fluid is given by

$E_{i}=\rho_{i} v$

where, $v=v_{0} a^{3}(t)$ is the physical volume of universe at given time $t$ and at present time $t_{0}$, the physical volume is $v_{0}$ with $a\left(t_{0}\right)=1$. According to the 1 st law of thermodynamics (which is an energy conservation equation),

$T d S_{i}=d E_{i}+p_{i} d v$.

Integrating Eq. (6) one can easily find the expression for energy density of the ith fluid as,

$\rho_{i}=a^{-3\left(1+\omega_{i}\right)}\left[\rho_{i 0}+\gamma_{i} \int_{t_{0}}^{t} a^{3 \omega_{i}} d t\right]$

where $\rho_{i 0}$ is the value of $\rho_{i}$ at $t=t_{0}$ i.e. at the present time .The energy density of ith fluid, from Eq. (9) can be arranged as

$d\left(\ln \rho_{i}\right)=-\left(1+\omega_{i}\right) d(\ln v)+d\left[\ln \left(\rho_{i 0}+\gamma_{i} A_{i}\right)\right]$

where $A_{i}(t)=\frac{1}{v_{0}} \int_{t_{0}}^{t} v^{\omega_{i}} d t$. Now, taking entropy $S_{i}$ as a function of two independent thermodynamic variables namely volume $(v)$ and temperature $(T)$ and also considering $d S_{i}$ to be an exact differential, one obtains from equation (8),

$d \ln T=-\omega_{i} d \ln v+\frac{w_{i}}{1+\omega_{i}} d\left[\ln \left(\rho_{i 0}+\gamma_{i} A_{i}\right)\right]$.

Now combining Eqs.(10) and (11), a new relation among energy density, temperature and time can be written as

$\frac{T}{\rho_{i} v}=\beta\left[\rho_{i 0}+\gamma_{i} A_{i}\right]^{-\frac{1}{1+\omega_{i}}}$,

where $\beta$ is an integration constant. Effectively at present time $t=t_{0}$,

$\frac{T_{0}}{\rho_{i 0} v_{0}}=\beta \rho_{i 0}^{-\frac{1}{1+\omega_{i}}}$.

Now Eqs. (12) and (13) imply the evolution of energy of the ith fluid with time and temperature as,

$E_{i}=E_{i 0}\left(\frac{T}{T_{0}}\right)\left[1+\frac{\gamma_{i}}{\rho_{i 0}} A_{i}\right]^{\frac{1}{1+\omega_{i}}}$

This can be termed as the modified equation of state of this fluid where as before $E_{i}=\rho_{i} v$ and $E_{i 0}=\rho_{i 0} v_{0}$. 
2.1 Determination of thermodynamic derivatives:

$$
C_{p}, C_{v}, K_{S}, K_{T}
$$

Now, the motivation of the present work is to find the criteria of thermodynamic stability of the universe. So the conditions which may be satisfied by the parameters, indicate the thermodynamic features of the evolution of the expanding universe. For any system the thermodynamic derivatives i.e. the heat capacities,compressibility and isobaric expansibility determine whether the system is stable or not. So it will be very important to analyze these parameters for each fluid in the present context.

From the 1st law of thermodynamics in Eq. (8), one can determine the two heat capacities: heat capacity at constant volume $C_{v}$ and heat capacity at constant pressure $C_{p}$ by the relations,

$$
\begin{aligned}
C_{v} & =\left[\frac{\partial Q}{\partial T}\right]_{v}=\left[\frac{\partial E}{\partial T}\right]_{v} \\
C_{p} & =\left[\frac{\partial Q}{\partial T}\right]_{p}=\left[\frac{\partial H}{\partial T}\right]_{p},
\end{aligned}
$$

where $H=E+p v$, is called the enthalpy of the system.

Now the relation between temperature of a system at a constant volume $\left(T_{a}\right)$ and the physical equilibrium temperature is given by (according to [42])

$T=T_{a}\left(1+\frac{\gamma_{i}}{\rho_{i 0}} A_{i}\right)$

The temperature at a constant volume $T_{a}$ also evolves as

$$
T_{a}=T_{0} a^{-3 \omega_{i}} .
$$

So from Eqs. (17) and (18),

$E_{i}=E_{i 0}\left(\frac{1}{T_{0}}\right) T^{\frac{2+\omega_{i}}{1+\omega_{i}}} T_{a}^{-\frac{1}{1+\omega_{i}}}$

Now from Eqs. (15) and (19) one obtains,

$C_{i v}=\frac{2+\omega_{i}}{1+\omega_{i}}\left(\frac{E_{i 0}}{T_{0}}\right)\left(\frac{T}{T_{a}}\right)^{\frac{1}{1+\omega_{i}}}$,

and Eqs. (1), (16) and (19) yield (using the definition of enthalpy)

$$
\begin{aligned}
C_{i p}= & \left(2+\omega_{i}\right)\left(\frac{E_{i 0}}{T_{0}}\right)\left(\frac{T}{T_{a}}\right)^{\frac{1}{1+\omega_{i}}} \\
& {\left[1-\frac{1}{2+\omega_{i}}\left(\frac{T}{T_{a}}\right)\left(\frac{\partial T_{a}}{\partial T}\right)_{p_{i}}\right] . }
\end{aligned}
$$

Also Eq. (21) can be rewritten (using 17) as

$$
\begin{aligned}
C_{i p}= & \frac{E_{0}}{T_{0}}\left(\frac{T}{T_{a}}\right)^{\frac{1}{1+\omega_{i}}} \\
& {\left[\left(1+\omega_{i}\right)+T\left\{\frac{\partial}{\partial T} \ln \left(1+\frac{\gamma_{i}}{\rho_{i 0}} A_{i}\right)\right\}_{p_{i}}\right] . }
\end{aligned}
$$

Further assuming temperature and pressure as independent thermodynamic variables, the variation of volume can be expressed as (See Refs. [34] and [35])

$d v=v\left(\alpha d T-K_{T} d p\right)$

where

$\alpha=\frac{1}{v}\left(\frac{\partial v}{\partial T}\right)_{p}$

is familiar as thermal expansibility. The isothermal compressibility $\left(K_{T}\right)$ is given by

$K_{T}=-\frac{1}{v}\left(\frac{\partial v}{\partial p}\right)_{T}$.

Similarly the adiabatic compressibility is

$K_{S}=-\frac{1}{v}\left(\frac{\partial v}{\partial p}\right)_{S}$.

In isothermal process,

$\frac{\alpha}{K_{T}}=\left(\frac{\partial p}{\partial T}\right)_{v}$.

Also one has the well established relation amongst heat capacities and compressibilities as [34]

$\frac{C_{p}}{C_{v}}=\frac{K_{S}}{K_{T}}$.

So one can find the expression for isothermal expansibility $\alpha$ from Eq.(11) as,

$\alpha_{i}=-\frac{1}{\omega_{i} T}+\frac{1}{1+\omega_{i}}\left[\frac{\partial}{\partial T}\left\{\ln \left(1+\frac{\gamma_{i}}{\rho_{i 0}} A_{i}\right)\right\}\right]_{p_{i}}$.

Now Eqs. (27) and (28) yield

$K_{i T}=\frac{\alpha_{i} v}{\omega_{i} C_{i v}}$

$K_{i S}=\frac{\alpha_{i} v}{\omega_{i} C_{i p}}$.

Hence one obtains the expressions for compressibilities from Eqs. (29), (30) and (31) containing the dependence of diffusion parameter $\gamma_{i}$ as,

$$
\begin{gathered}
K_{i T}=\frac{v}{\omega_{i} C_{i v}}\left[-\frac{1}{\omega_{i} T}+\frac{1}{1+\omega_{i}}\left\{\frac{\partial}{\partial T} \ln \left(1+\frac{\gamma_{i}}{\rho_{i 0}} A_{i}\right)\right\}_{p_{i}}\right] \\
K_{i S}=\frac{v}{\omega_{i} C_{i p}}\left[-\frac{1}{\omega_{i} T}+\frac{1}{1+\omega_{i}}\left\{\frac{\partial}{\partial T} \ln \left(1+\frac{\gamma_{i}}{\rho_{i 0}} A_{i}\right)\right\}_{p_{i}}\right] .
\end{gathered}
$$




\subsection{Stability conditions for cosmic fluids}

For thermodynamic stability of any fluid, it must follow the conditions [34,35] namely $C_{p}, C_{v}, K_{T}, K_{S} \geq 0$. For the present work, using the expressions of these thermodynamical parameters, the stability conditions are presented in the following Table 1:

\section{Stability criteria with variable equation of state parameter}

So far we have examined the conditions for stability of cosmic fluids with constant equation of state parameters. In the present section the stability conditions will be discussed for cosmic fluids having variable equation of state parameters . The fluids are diffusive as before with no interaction amongst them. In such context, the solution of the conservation Eq. (6) yields,

$\rho_{i}=\left[\rho_{i 0}+\gamma_{i} \int_{t_{0}}^{t} a^{-3} e^{F_{i}(a)} d t\right] e^{-F_{i}(a)}$

where,

$F_{i}(a)=\int\left(1+\omega_{i}\right) d(\ln v)$

Taking

$\int_{t_{0}}^{t} a^{-3} e^{F_{i}(a)} d t=A_{i}(t)$,

one can write Eq. (34) as

$\rho_{i}=e^{-F_{i}(a)}\left[\rho_{i 0}+\gamma_{i} A_{i}(t)\right]$.

3.1 Derivation of the relation between physical equilibrium temperature $(\mathrm{T})$ and temperature at constant volume $\left(T_{a}\right)$

From 1st law of thermodynamics (i.e. Eq. (8)), by choosing entropy (S) as a function of two independent variables volume (v) and temperature (T), the condition for dS to be an exact differential gives

$$
\begin{aligned}
\left(\frac{\partial S}{\partial v}\right)_{T} & =\frac{\rho}{T}(1+\omega) \\
\left(\frac{\partial S}{\partial T}\right)_{v} & =\frac{v}{T} \frac{d \rho}{d T} \\
\text { and } \frac{d \rho}{\rho} & =\frac{1+\omega}{\omega} \frac{d T}{T}-\frac{d \omega}{\omega} .
\end{aligned}
$$

Now, from Eq. (39) one obtains the energy density as a function of temperature as

$\rho=\frac{B}{\omega} e^{\int \frac{1+\omega}{\omega} \frac{d T}{T}}$.
Again, combining Eqs. (38) and (40) the expression for entropy can be written as,

$S=v \frac{1+\omega}{\omega T_{a}} B e^{\int \frac{1+\omega}{\omega} \frac{d T_{a}}{T_{a}}}$.

Now, as for an adiabatic system $\Delta S=0$, so one can determine from Eq. (41), the relation between $T_{0}$ and $T_{a}$ (i.e. evolution of $T_{a}$ ) as

$T_{a} e^{-\int_{\text {present }}^{t} \frac{1+\omega}{\omega} \frac{d T_{a}}{T_{a}}}=T_{0} \frac{1+\omega}{\omega} \frac{\omega_{0}}{1+\omega_{0}} a^{3}$.

Now combining Eqs. (35), (36) and (42) one obtains,

$\rho_{i}=\frac{1}{\omega_{i}} e^{\int_{\text {present }}^{t} \frac{1+\omega_{i}}{\omega_{i}} \frac{d T_{a}}{T_{a}}}\left[\rho_{i 0}+\gamma_{i} A_{i}(t)\right]$

or equivalently

$\rho_{i}=\frac{1}{\omega_{i}} e^{\int_{\text {present }}^{t} \frac{1+\omega_{i}}{\omega_{i}} \frac{d T_{a}}{T_{a}}} 3 z_{i} B\left(A_{i}\right) v_{0}^{-1}$

where $z_{i}=\frac{\rho_{i 0} v_{0}}{3 T_{0}}$ and $B\left(A_{i}\right)=T_{0}+\frac{\gamma_{i} T_{0}}{\rho_{i 0}} A_{i}(t)$.

Now as the adiabatic contribution of entropy for the ith fluid (excluding the diffusive entropy $\left(S_{i d}=\int \frac{v_{o} \gamma_{i}}{T} d T\right)$ ) namely,

$S_{i(a d)}=\frac{\left(1+\omega_{i}\right) \rho_{i} v}{T}$

does not vary (i.e. $d \ln \left(S_{i(a d)}\right)=0$ ), so temperature can be written as,

$T=\frac{1+\omega_{i 0}}{\omega_{i 0} L}\left(\frac{T_{a}}{T_{0}}\right) B\left(A_{i}\right)$

where, $L$ is a constant . Now combining (45) and (46) yields

$$
\left(1+\frac{\gamma_{i}}{\rho_{i 0}} A_{i}\right)=\left(\frac{T}{T_{a}}\right) Q
$$

with $Q=\frac{\omega_{i 0} L}{1+\omega_{i 0}}$, a constant .

\subsection{Thermodynamic derivatives}

Similar to the above section, one can determine the heat capacities and compressibilities of the present thermodynamical system as follows: now the solution of Eq. (6), (or equivalently Eq. (34)) can be expressed as

$d \ln \rho_{i}=-\left(1+\omega_{i}\right) d \ln v+d \ln \left[1+\frac{\gamma_{i}}{\rho i 0} A_{i}\right]$.

Again from Eq. (39), one finds

$d \ln \rho_{i}=\frac{1+\omega_{i}}{\omega_{i}} d \ln T-\frac{d \omega}{\omega}$

Combining the above Eqs. (48) and (49) one can write,

$\frac{T}{E_{i}\left(1+\omega_{i}\right)}=C e^{-\int \frac{1}{1+\omega_{i}} d \ln \left[1+\frac{\gamma_{i}}{\rho_{i} 0} A_{i}\right]}$. 
Table 1 Conditions for stability

\begin{tabular}{ll}
\hline Range of $\omega_{i}$ & Stability condition \\
\hline$\omega_{i} \geq 0$ & {$\left[\frac{\partial\left\{\ln \left(1+\frac{\gamma_{i}}{\rho_{i} 0} A_{i}\right)\right\}}{\partial \ln T}\right]_{p_{i}} \geq \frac{1+\omega_{i}}{\omega_{i}}$} \\
$-1 \leq \omega_{i} \leq 0$ & $-\left(1+\omega_{i}\right) \leq\left[\frac{\partial\left\{\ln \left(1+\frac{\gamma_{i}}{\rho_{i} 0} A_{i}\right)\right\}}{\partial \ln T}\right]_{p_{i}}$ and $\left[\frac{\partial\left\{\ln \left(1+\frac{\gamma_{i}}{\rho_{i} 0} A_{i}\right)\right\}}{\partial \ln T}\right]_{p_{i}} \leq \frac{1+\omega_{i}}{\omega_{i}}$ simultaneously, which is not possible hence unstable \\
$-2 \leq \omega_{i} \leq-1$ & Unstable \\
$\omega_{i} \leq-2$ & {$\left[\frac{\partial\left\{\ln \left(1+\frac{\gamma_{i}}{\rho_{i} 0} A_{i}\right)\right\}}{\partial \ln T}\right]_{p_{i}} \geq-\left(1+\omega_{i}\right)$} \\
\hline
\end{tabular}

Table 2 Conditions for stability criteria

\begin{tabular}{ll}
\hline Range of $\omega_{i}$ & Stability condition \\
\hline$\omega_{i} \geq 0$ & $\frac{d \ln \omega_{i}}{d \ln T} \leq \frac{2+\omega_{i}}{\omega_{i}}$ and $\left[\frac{\partial\left\{\ln \left(1+\frac{\gamma_{i}}{\rho_{i} 0} A_{i}\right)\right\}}{\partial \ln T}\right]_{p_{i}} \geq \max \left[-\left(1+\omega_{i}\right),\left(\frac{1+\omega_{i}}{\omega_{i}}-\frac{d \ln \omega_{i}}{d \ln T}\right)\right]$ \\
$-1 \leq \omega_{i} \leq 0$ & $\frac{d \ln \omega_{i}}{d \ln T} \geq \frac{2+\omega_{i}}{\omega_{i}}$ and $\left[\frac{\partial\left\{\ln \left(1+\frac{\gamma_{i}}{\rho_{i} 0} A_{i}\right)\right\}}{\partial \ln T}\right]_{p_{i}} \geq \max \left[-\left(1+\omega_{i}\right),\left(\frac{1+\omega_{i}}{\omega_{i}}-\frac{d \ln \omega_{i}}{d \ln T}\right)\right]$ if $\frac{d \ln \omega_{i}}{d \ln T} \leq$ \\
$\omega_{i} \leq-1$ & $\frac{1+3 \omega_{i}+2 \omega_{i}^{2}}{\omega_{i}^{2}} \cdot \operatorname{Again}-\left(1+\omega_{i}\right) \leq\left[\frac{\partial\left\{\ln \left(1+\frac{\gamma_{i}}{\rho_{i} 0} A_{i}\right)\right\}}{\partial \ln T}\right]_{p_{i}} \leq\left(\frac{1+\omega_{i}}{\omega_{i}}-\frac{d \ln \omega_{i}}{d \ln T}\right) \operatorname{if} \frac{d \ln \omega_{i}}{d \ln T} \geq \frac{1+3 \omega_{i}+2 \omega_{i}^{2}}{\omega_{i}^{2}}$ \\
\hline & $\frac{d \ln \omega_{i}}{d \ln T} \leq \frac{2+\omega_{i}}{\omega_{i}}$ and $\left[\frac{\partial\left\{\ln \left(1+\frac{\gamma_{i}}{\rho_{i} 0} A_{i}\right)\right\}}{\partial \ln T}\right]_{p_{i}} \geq \max \left[-\left(1+\omega_{i}\right),\left(\frac{1+\omega_{i}}{\omega_{i}}-\frac{d \ln \omega_{i}}{d \ln T}\right)\right]$
\end{tabular}

Now imposing the initial conditions, one can formulate the modified equation of state in terms of evolution of energy with time and temperature as

$E_{i}=E_{i 0}\left(\frac{1+\omega_{i 0}}{1+\omega_{i}}\right)\left(\frac{T}{T_{0}}\right) e^{\int_{\text {present }}^{t} \frac{1}{1+\omega_{i}} d \ln \left(1+\frac{\gamma_{i}}{\rho_{i 0}} A_{i}\right)}$.

The above Eq. (51) can be written according to Eq. (47) as

$E_{i}=E_{i 0}\left(\frac{1+\omega_{i 0}}{1+\omega_{i}}\right)\left(\frac{T}{T_{0}}\right) e^{\int_{\text {present }}^{t} \frac{1}{1+\omega_{i}} d \ln \left(\frac{T}{T_{a}}\right)}$.

So using Eqs. (15), (16), (47) and (51), one can easily express the heat capacities as

$$
\begin{aligned}
C_{i v} & =\frac{E_{i}}{T\left(1+\omega_{i}\right)}\left[\left(2+\omega_{i}\right)-T \frac{d \omega}{d T}\right] \\
C_{i p} & =\frac{E_{i}}{T}\left[\left(1+\omega_{i}\right)+T\left\{\frac{\partial}{\partial T} \ln \left(1+\frac{\gamma_{i}}{\rho_{i 0}} A_{i}\right)\right\}_{p}\right]
\end{aligned}
$$

and hence one obtains the relation

$$
\left(\frac{\partial p}{\partial T}\right)_{v}=\frac{\omega}{v} C_{v}+\rho \frac{d \omega}{d T}
$$

Again Eqs. (48) and (49) yield

$d \ln T=\frac{d \omega_{i}}{1+\omega_{i}}-\omega_{i} d \ln v+\frac{\omega_{i}}{1+\omega_{i}} d \ln \left(1+\frac{\gamma_{i}}{\rho_{i 0}} A_{i}\right)$,

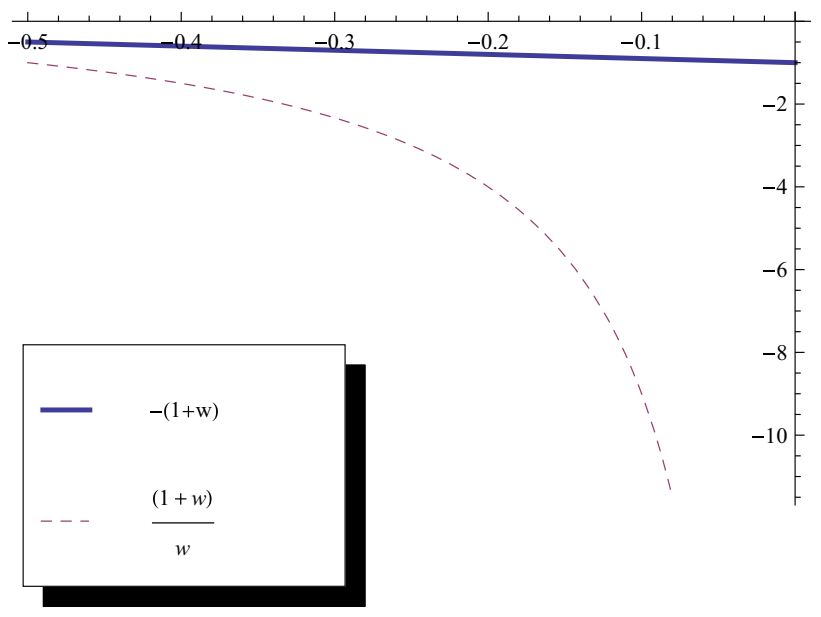

Fig. 1 No point having value greater than $-\left(1+\omega_{i}\right)$ and less than $\frac{1+\omega_{i}}{\omega_{i}}$ simultaneously

which implies along with Eq. (47) that

$$
\left(\frac{\partial \omega}{\partial T}\right)_{v}=\frac{1+\omega_{i}+\omega_{i}^{2}}{T\left(1+\omega_{i}\right)} \text {. }
$$

So from Eqs. (27), (53), (55) and (57) one writes

$$
K_{i T}=\frac{\alpha_{i} v\left[2+\omega_{i}-T \frac{d \omega_{i}}{d T}\right]}{C_{i v}\left[\omega_{i}\left\{1+\left(2+\omega_{i}-T \frac{d \omega_{i}}{d T}\right)\right\}+1+\omega_{i}^{2}\right]}
$$




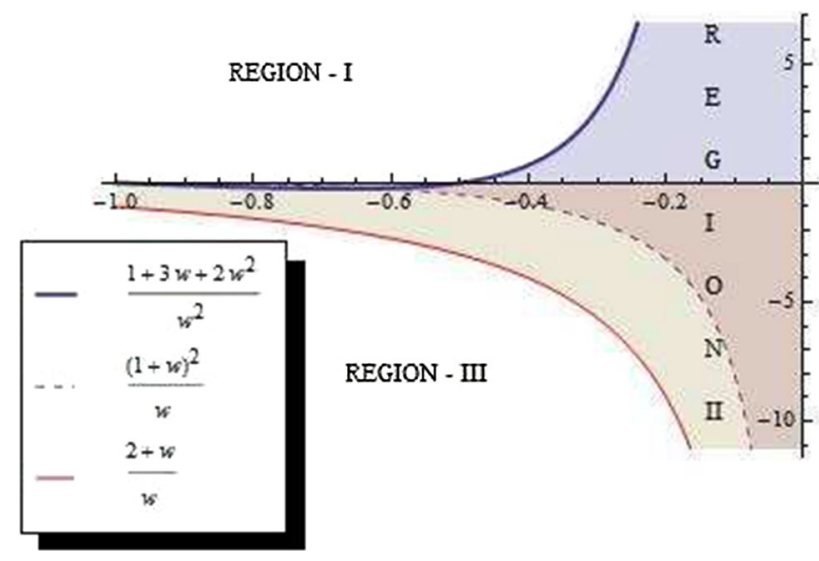

Fig. 2 For the values of $\frac{d \ln \omega_{i}}{d \ln T} \in$ REGION-I, the stability condition is $-\left(1+\omega_{i}\right) \leq\left[\frac{\partial\left\{\ln \left(1+\frac{\gamma_{i}}{\rho_{i} 0} A_{i}\right)\right\}}{\partial \ln T}\right]_{p_{i}} \leq\left(\frac{1+\omega_{i}}{\omega_{i}}-\frac{d \ln \omega_{i}}{d \ln T}\right)$, for $\frac{d \ln \omega_{i}}{d \ln T} \epsilon$ REGION-II, the stability condition is $\left[\frac{\partial\left\{\ln \left(1+\frac{\gamma_{i}}{\rho_{i 0}} A_{i}\right)\right\}}{\partial \ln T}\right]_{p_{i}} \geq$ $\max \left[-\left(1+\omega_{i}\right),\left(\frac{1+\omega_{i}}{\omega_{i}}-\frac{d \ln \omega_{i}}{d \ln T}\right)\right]$ and in REGION-III unstable

and from (28), one obtains

$K_{i S}=\frac{\alpha_{i} v\left[2+\omega_{i}-T \frac{d \omega_{i}}{d T}\right]}{C_{i p}\left[\omega_{i}\left\{1+\left(2+\omega_{i}-T \frac{d \omega_{i}}{d T}\right)\right\}+1+\omega_{i}^{2}\right]}$.

Now, using Eq. (56) the expression for $\alpha_{i}$ takes the form

$$
\begin{aligned}
\alpha_{i}= & {\left[\frac{\partial(\ln V)}{\partial T}\right]_{p_{i}}=\frac{1}{\omega_{i}\left(1+\omega_{i}\right)}\left[\omega_{i}\left\{\frac{\partial}{\partial T} \ln \left(1+\frac{\gamma_{i}}{\rho_{i 0}} A_{i}\right)\right\}_{p_{i}}\right.} \\
& \left.+\frac{d \omega_{i}}{d T}-\frac{1+\omega_{i}}{T}\right]
\end{aligned}
$$

and the expressions for $K_{T}$ and $K_{S}$ are given by (using Eqs. (58), (59) and (60))

$$
K_{i T}=\frac{V\left[\omega_{i}\left\{\frac{\partial}{\partial T} \ln \left(1+\frac{\gamma_{i}}{\rho_{i 0}} A_{i}\right)\right\}_{p_{i}}+\frac{d \omega_{i}}{d T}-\frac{1+\omega_{i}}{T}\right]\left[2+\omega_{i}-T \frac{d \omega_{i}}{d T}\right]}{\omega_{i}\left(1+\omega_{i}\right) C_{i v}\left[\omega_{i}\left\{1+\left(2+\omega_{i}-T \frac{d \omega_{i}}{d T}\right)\right\}+1+\omega_{i}^{2}\right]}
$$

and $K_{i S}=\frac{V\left[\omega_{i}\left\{\frac{\partial}{\partial_{T}} \ln \left(1+\frac{\gamma_{i}}{\rho_{i 0}} A_{i}\right)\right\}_{p_{i}}+\frac{d \omega_{i}}{d T}-\frac{1+\omega_{i}}{T}\right]\left[2+\omega_{i}-T \frac{d \omega_{i}}{d T}\right]}{\omega_{i}\left(1+\omega_{i}\right) C_{i p}\left[\omega_{i}\left\{1+\left(2+\omega_{i}-T \frac{d \omega_{i}}{d T}\right)\right\}+1+\omega_{i}^{2}\right]}$.

Now according to the conditions of thermodynamic stability, we analyze the Eqs. (53), (54), (61) and (62) to find the restrictions of thermodynamic stability in different ranges of $\omega_{i}$ in the following Table 2.

\section{Brief discussions}

A detailed thermodynamic study of non-interacting cosmic fluids having diffusive nature has been organized in the present work. As a result, individual fluid does not obey the energy conservation relation, rather the non-conservation is proportional to the corresponding diffusive current. Each component of the cosmic fluids is assumed to have barotropic equation of state with constant or variable equation of state parameter. The stability conditions are expressed in the form of inequalities for different ranges of equation of state parameter. Also, the stability conditions for both constant (Table 1) and variable (Table 2) equation of state parameter in the range $-1<\omega_{i}<0$ (i.e. for dark energy region) have been presented graphically in Figs. 1 and 2 respectively. From Fig. 2, different restrictions on diffusion parameter $\gamma_{i}$ are found depending on whether the values of $\frac{d \ln \omega_{i}}{d \ln T}$ (i.e. relative variation of $\omega_{i}$ with respect to the temperature T) belong to REGION-I or REGION-II . However due to expanding nature of the universe, the isobaric expansibility $\left(\alpha_{i}\right)$ should always be positive. This condition restricts the function $\frac{d \ln \omega_{i}}{d \ln T}$ only in REGION-II, which is acceptable for expanding universe (Table 3).

Further, it is interesting to note that, for constant equation of state parameter, (i.e. Table 1), the system cannot be thermodynamically stable for $-1<\omega_{i} \leq 0$. This result is very much similar to that of [34]. However, the present work is thermodynamically stable in phantom region in contrary with non-diffusive cosmic fluids [34]. Finally, it is found that diffusive fluid with variable equation of state parameter is

Table 3 Conclusion on the result in dark energy range $\left(-0.33<\omega_{i}<0\right)$

\begin{tabular}{lll}
\hline Nature of dark energy & $\begin{array}{l}\text { Constraint on } \\
\text { diffusion } \\
\text { parameter } \gamma_{i}\end{array}$ & Restriction on $\frac{d \ln \omega_{i}}{d \ln T}$ from graphs \\
\hline Constant equation of state parameter & $\begin{array}{l}\text { Always unstable } \\
\text { Variable equation of state parameter }\end{array}$ & {$\left[\frac{\partial\left\{\ln \left(1+\frac{\gamma_{i}}{\rho_{i} 0} A_{i}\right)\right\}}{\partial \ln T}\right]_{p_{i}} \geq$} \\
& $\max \left[-\left(1+\omega_{i}\right),\left(\frac{1+\omega_{i}}{\omega_{i}}-\frac{d \ln \omega_{i}}{d \ln T}\right)\right]$ & $\frac{2+\omega_{i}}{\omega_{i}} \leq \frac{d \ln \omega_{i}}{d \ln T} \leq \frac{1+3 \omega_{i}+2 \omega_{i}^{2}}{\omega_{i}^{2}}$ \\
\hline
\end{tabular}


thermodynamically stable for all possible values of $\omega_{i}$ under some restrictions on the diffusion parameter $\gamma_{i}$ and nature of variation of $\omega_{i}$ with respect to temperature.

Acknowledgements The authors are thankful to IUCAA, Pune, India for research facilities at Library. The author SM acknowledges UGCJRF and PB acknowledges DST-INSPIRE (File no: IF160086) for awarding Research fellowship. Also SC acknowledges the UGC-DRS Program in the Department of Mathematics, Jadavpur University.

Data Availability Statement This manuscript has no associated data or the data will not be deposited. [Authors' comment: We have not use any real astronomical data in our paper.]

Open Access This article is distributed under the terms of the Creative Commons Attribution 4.0 International License (http://creativecomm ons.org/licenses/by/4.0/), which permits unrestricted use, distribution, and reproduction in any medium, provided you give appropriate credit to the original author(s) and the source, provide a link to the Creative Commons license, and indicate if changes were made. Funded by SCOAP 3 .

\section{References}

1. S. Perlmutter et al., [Supernova Cosmology Project Collaboration], Measurements of Omega and Lambda from 42 high redshift supernovae. Astrophys. J. 517, 565 (1999). arXiv:astro-ph/9812133

2. A.G. Riess et al., [Supernova Search Team], Observational evidence from supernovae for an accelerating universe and a cosmological constant. Astron. J. 116, 1009 (1998). arXiv:astro-ph/9805201

3. P. de Bernardis et al., [Boomerang Collaboration], A Flat universe from high resolution maps of the cosmic microwave background radiation. Nature 404, 955 (2000). arXiv:astro-ph/0004404

4. W.J. Percival et al., [2dFGRS Collaboration], The 2dF Galaxy Redshift Survey: The Power spectrum and the matter content of the Universe. Mon. Not. Roy. Astron. Soc. 327, 1297 (2001). arXiv:astro-ph/0105252

5. D.N. Spergel et al., [WMAP Collaboration], First year Wilkinson Microwave Anisotropy Probe (WMAP) observations : Determination of cosmological parameters. Astrophys. J. Suppl. 148, 175 (2003). arXiv:astro-ph/0302209

6. B. Jain, A. Taylor, Cross-correlation tomography: measuring dark energy evolution with weak lensing. Phys. Rev. Lett. 91, 141302 (2003). arXiv:astro-ph/0306046

7. M. Tegmark et al., [SDSS Collaboration], Cosmological parameters from SDSS and WMAP. Phys. Rev. D 69, 103501 (2004). arXiv:astro-ph/0310723

8. D.J. Eisenstein et al., [SDSS Collaboration], Detection of the Baryon Acoustic Peak in the Large-Scale Correlation Function of SDSS Luminous Red Galaxies. Astrophys. J. 633, 560 (2005). arXiv:astro-ph/0501171

9. E. Komatsu et al., [WMAP Collaboration], Seven-Year Wilkinson Microwave Anisotropy Probe (WMAP) Observations: Cosmological Interpretation, Astrophys. J. Suppl. 192, 18(2011). arXiv: 1001.4538 [astro-ph.CO]

10. P. A. R. Ade et al., [Planck Collaboration], Planck 2015 results. XIII. Cosmological parameters, Astron. Astrophys. 594, A13(2016). arXiv:1502.01589 [astro-ph.CO]

11. S. Weinberg, The cosmological constant problem. Rev. Mod. Phys. 61, 1 (1989)

12. T. Padmanabhan, Cosmological constant: the weight of the vacuum. Phys. Rept. 380, 235 (2003). arXiv:hep-th/0212290
13. P.J. Steinhardt, A quintessential introduction to dark energy. Philos. Trans. R. Soc. Lond. A 361, 2497 (2003)

14. L. Amendola, S. Tsujikawa, Dark energy: theory and observations (Cambridge University Press, Cambridge, 2010)

15. Y.L. Bolotin, A. Kostenko, O.A. Lemets, D.A. Yerokhin, Cosmological evolution with interaction between dark energy and dark matter. Int. J. Mod. Phys. D 24(03), 1530007 (2014). arXiv:1310.0085 [astro-ph.CO]

16. B. Wang, E. Abdalla, F. Atrio-Barandela, D. Pavon, Dark matter and dark energy interactions: theoretical challenges, cosmological implications and observational signaturesD. Rept. Prog. Phys. 79(9), 096901 (2016). arXiv:1603.08299 [astro-ph.CO]

17. G.S. Sharov, S. Bhattacharya, S. Pan, R.C. Nunes, S. Chakraborty, A new interacting two fluid model and its consequences. Mon. Not. Roy. Astron. Soc. 466(3), 3497 (2017). arXiv:1701.00780 [gr-qc]

18. V. Salvatelli, N. Said, M. Bruni, A. Melchiorri, D. Wands, Indications of a late-time interaction in the dark sector. Phys. Rev. Lett. 113(18), 181301 (2014). arXiv:1406.7297 [astro-ph.CO]

19. J. Sola, A. Gomez-Valent, J. de Cruz Prez, Hints of dynamical vacuum energy in the expanding Universe, Astrophys. J. 811, L14 (2015). arXiv:1506.05793 [gr-qc]

20. J. Sola, J. de Cruz Prez, A. Gomez-Valent, R.C. Nunes, Dynamical Vacuum against a rigid Cosmological Constant. arXiv:1606.00450 [gr-qc]

21. R.C. Nunes, S. Pan, E.N. Saridakis, New constraints on interacting dark energy from cosmic chronometers. Phys. Rev. D 94(2), 023508 (2016). arXiv:1605.01712 [astro-ph.CO]

22. S. Kumar, R.C. Nunes, Probing the interaction between dark matter and dark energy in the presence of massive neutrinos. Phys. Rev. D 94(12), 123511 (2016). arXiv:1608.02454 [astro-ph.CO]

23. C. van de Bruck, J. Mifsud, J. Morrice, Testing coupled dark energy models with their cosmological background evolution. Phys. Rev. D 95(4), 043513 (2017). arXiv:1609.09855 [astro-ph.CO]

24. T. Yang, Z.K. Guo, R.G. Cai, Reconstructing the interaction between dark energy and dark matter using Gaussian Processes. Phys. Rev. D 91(12), 123533 (2015). arXiv:1505.04443 [astroph.CO]

25. W. Yang, L. Xu, Cosmological constraints on interacting dark energy with redshift-space distortion after Planck data. Phys. Rev. D 89(8), 083517 (2014). arXiv:1401.1286 [astro-ph.CO]

26. W. Yang, L. Xu, Coupled dark energy with perturbed Hubble expansion rate. Phys. Rev. D 90(8), 083532 (2014). arXiv:1409.5533 [astro-ph.CO]

27. W. Yang, H. Li, Y. Wu, J. Lu, Cosmological constraints on coupled dark energy. JCAP 1610(10), 007 (2016). arXiv:1608.07039 [astroph.CO]

28. D.M. Xia, S. Wang, Constraining interacting dark energy models with latest cosmological observations. Mon. Not. Roy. Astron. Soc. 463(1), 952 (2016). arXiv: 1608.04545 [astro-ph.CO]

29. C. Caprini, N. Tamanini, Constraining early and interacting dark energy with gravitational wave standard sirens: the potential of the eLISA mission. JCAP 1610(10), 006 (2016). arXiv:1607.08755 [astro-ph.CO]

30. R. Murgia, S. Gariazzo, N. Fornengo, Constraints on the coupling between dark energy and dark matter from CMB data. JCAP 1604(04), 014 (2016). arXiv:1602.01765 [astro-ph.CO]

31. A. Pourtsidou, T. Tram, Reconciling CMB and structure growth measurements with dark energy interactions. Phys. Rev. D 94(4), 043518 (2016). arXiv: 1604.04222 [astro-ph.CO]

32. W. Zimdahl, Interacting dark energy and cosmological equations of state. Int. J. Mod. Phys. D 14, 2319 (2005). arXiv:gr-qc/0505056

33. B. Wang, J. Zang, C.Y. Lin, E. Abdalla, S. Micheletti, Interacting dark energy and dark matter: observational constraints from cosmological parameters. Nucl. Phys. B 778, 69 (2007). arXiv:astro-ph/0607126 
34. E.M. Barboza, R.C. Nunes, E.M.C. Abreu, J.A. Neto, Thermodynamic aspects of dark energy fluids. Phys. Rev. D 92(8), 083526 (2015)

35. P. Bhandari, S. Haldar, S. Chakraborty, Interacting dark energy model and thermal stability. Eur. Phys. J. C 77(12), 840 (2017)

36. P. Bhandari, S. Haldar, S. Chakraborty, Thermodynamics of the dissipative cosmic fluid and stability criteria. Eur. Phys. J. A 54(5), 78 (2018). arXiv: 1806.00085 [gr-qc]

37. A.A. Mamon, P. Bhandari, S. Chakraborty, Study of thermal stability for different dark energy models. arXiv:1802.07925 [gr-qc]

38. S. Haldar, P. Bhandari, S. Chakraborty, Emergent Scenario in first and second order non-equilibrium thermodynamics and stability analysis. Annals Phys. 387, 203 (2017). arXiv:1710.06793 [gr-qc]
39. L. Amendola, G Camargo Campos, R. Rosenfeld, Consequences of dark matter-dark energy interaction on cosmological parameters derived from SNIa data. Phys. Rev. D 75, 083506 (2007)

40. C. Boehmer, G. Caldera-Cabral, R. Lazkoz, R. Maartens, Dynamics of dark energy with a coupling to dark matter. AIP Conf. Proc. 1122, 197 (2009)

41. J.A.S. Lima, I. Baranov, Gravitationally induced particle production: thermodynamics and kinetic theory. Phys. Rev. D 90(4), 043515 (2014). arXiv: 1411.6589 [gr-qc]

42. Z. Haba, Thermodynamics of diffusive DM/DE systems. Gen. Rel. Grav. 49(4), 58 (2017). arXiv:1612.05999 [gr-qc] 